\author{
EVS24 \\ Stavanger, Norway, May 13-16, 2009
}

\title{
Front-and-Rear-Wheel-Independent-Drive-Type Electric Vehicle (FRID EV) with Compatible Driving Performance and Safety
}

\author{
Nobuyoshi Mutoh ${ }^{1}$ \\ ${ }^{1}$ Graduate School of Tokyo Metropolitan University \\ 6-6, Asahigaoka, Hino-shi, Tokyo, 191-0065 Japan \\ nmutoh@cc.tmit.ac.jp
}

\begin{abstract}
This paper focuses on a front-and-rear-wheel-independent-drive-type-electric vehicle (FRID EV) which is being developed as the next generation EV with ideal characteristics of driving performance and safety. The FRID EV development work has included studying the driving performance and safety of all types of propulsion systems. Since the FRID EV has propulsion systems which can control the driving and braking torques of the front and rear wheels independently, various outstanding and indispensable functions are produced. Three functions which EVs developed until now could not have are shown here analytically and experimentally. The first is the failsafe function under which vehicles continue running without any unexpected sudden stops, even if one of the propulsion systems fails. The second is the function for performing efficient acceleration and deceleration on all roads by suitably distributing the driving or braking torques to the front and rear wheels according to the road surface conditions. The third is the function for ensuring safe running on bad roads, i. e., low $\mu$-roads like frozen roads, by simultaneously controlling slip ratios of the front and rear wheels in consideration of load movement. These are functions are unique to the FRID EV and they have not be realized previously in any gasoline engine-powered car, hybrid car or conventional electric vehicle.
\end{abstract}

Keywords: safety, EV (electric vehicle), lithium battery, electric drive, control system, diagnosis, motor, vehicle performance, braking, BEV (battery electric vehicle), failsafe, slip, front and rear wheels.

\section{Introduction}

More than 100 years have passed since the birth of the car, and the automobile industry is now facing very difficult problems stemming from such issues as global warming and dwindling fossil fuel resources. In order to cope with these problems, research and development of the next-generation vehicles is being furthered from different angles [1]. Various studies on electric vehicles (EVs) have been done [2]-[4]. In EVs, the safety and drive performance during running are influenced by the structure of the drive systems which generate propulsion force. The drive systems consist of many electronic 
parts, and failure of these parts may occur during running [5] [6]. When an unexpected sudden stop occurs due to this failure, there is a possibility a serious traffic accident may be caused. Thus, a failsafe structure by which the safety at the time of the failure during running is collateralized is indispensable to EVs. In addition, the drive structure needs to be constituted so that the sufficient drive performance can be obtained It has become clear that the driving structure of conventional EVs (Figs.1 (a), (b)) cannot meet the above demand. For example, in the one-motor drive type EVs (Fig.1(a)) [7][8], failure of drive systems induces a sudden stop, while, when a drive system of a left or right driving wheel fails, the torque centering on the failed driving wheel occurs, and a rear-end collision may be caused. Then, a front-and-rear-wheelindependent-drive-type electric vehicle (FIRD EV) (Fig.2) [9][10] with which the safety at the time of failure and the drive performance are compatible has been proposed [11]-[14]. Here, the outstanding drive performance which this FRID EV has is verified by simulations and a running test using a prototype FRID EV with practical specifications.

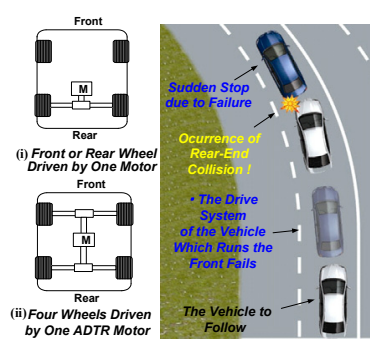

(a) One motor drive type EVs

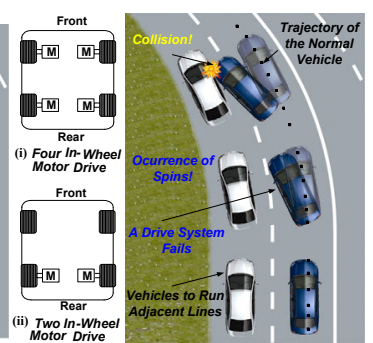

(b) In-wheel motor drive type EVs
Figure1: Drive system structure of conventional EVs and problems occurring when they fail.

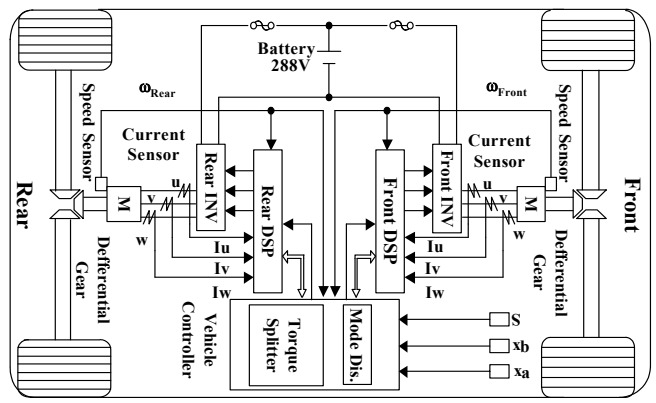

Figure2: FRID EV with the failsafe structure proposed by Mutoh et al. [11].

\section{Driving and Braking Torue Distributing Methods for the FRID EVs under Various Running Conditions}

\subsection{Driving Torque Distribution Method on Dry Roads}

The FRID EV has the structure which can distribute the driving forces to the front and rear wheel drives according to the running conditions. Fig.3 shows a driving force distribution method when accelerating on dry roads. Since load movement occurs to the rear wheels from the front wheels while accelerating, driving force is distributed to front and rear wheels in consideration of this load movement $z$ given by

$$
\begin{aligned}
& z=\left|\frac{F_{c a r} \cdot H_{c a r}}{L_{c a r}}\right| \\
& F_{c a r}=M_{c a r} \cdot \alpha_{\text {sensor }} .
\end{aligned}
$$

Here, $M_{c a r}$ is vehicle weight estimated using the method proposed by Mutoh et al. [14], $\alpha_{\text {sensor }}$ is a value obtained from an acceleration sensor, and $H_{c a r}$ is height of a center of gravity. Using the load movement calculated from (1), the front and rear driving torques, $\tau_{A f}{ }^{*}$ and $\tau_{A r}{ }^{*}$ distributed to the front and rear wheels, are expressed by

$$
\begin{gathered}
\tau_{A f} *=R_{f} \cdot \tau_{A}{ }^{*}, \\
\tau_{A r} *=R_{r} \cdot \tau_{A}{ }^{*},
\end{gathered}
$$

and

$$
R_{r}=\frac{F_{r}}{F_{f}+F_{r}}=\frac{\left(M_{c a r} / 2\right) \cdot g+z}{M_{c a r} \cdot g}, R_{f}=1-R_{r} .
$$

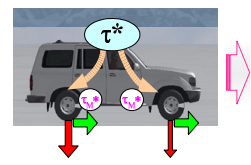
(a)Start to accelerate
(b) Compute load movement

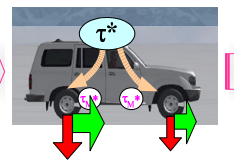

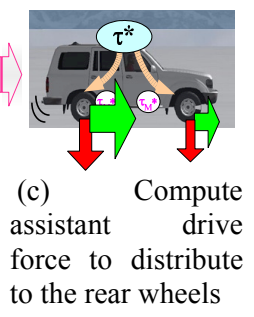

Figure 3: Driving torque distributing method when accelerating on dry roads. 


\subsection{Braking Torque Distribution Method on Dry Roads}

When braking is done during acceleration (Fig 4(a)), when releasing the accelerator (Fig. 4(b)), load movement occurs to the direction opposite that of movement, i.e., to the front wheels from the rear wheels (Fig. 4(c)). Therefore, when braking is done, the braking torque distributed to the front wheels is increased by the amount of the load movement; that is, the braking torques, $\tau_{B f}{ }^{*}$ and $\tau_{B r}{ }^{*}$ which are distributed to the front and rear wheels are given by

$$
\begin{aligned}
& \tau_{B f} *=R_{f} \cdot \tau_{B} *, \\
& \tau_{B r} *=R_{r} \cdot \tau_{B} *,
\end{aligned}
$$

and

$$
R_{r}=\frac{F_{r}}{F_{f}+F_{r}}=\frac{\left(M_{c a r} / 2\right) \cdot g-z}{M_{c a r} \cdot g}, R_{f}=1-R_{r} .
$$

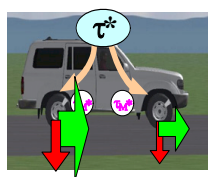

(a) Move load to the wheels during acceleration

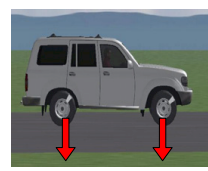

(b) Apply load rear uniformly to the front and rear wheels when the accelerator is released

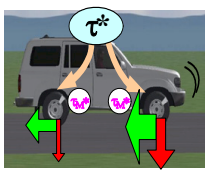

(c) Move load to the front wheels during braking
Figure 4: Braking torque distributing method when decelerating on dry roads.

\subsection{Driving Torque Distribution Method on Low Friction Coefficient Roads}

In order to run on a low friction coefficient $\mu$ road surface stably, it is necessary to generate driving force suitable for the road surface state between a tire and the road surface. Fig. 5 shows the stable domains of the slip ratio on various road surfaces in driving and braking regions [15]. The figure confirms that in driving regions the slip ratio for stable driving control is from 0.1 to 0.3 , even if the road surface changes from a dry road to a frozen road. Especially, on a low $\mu$ road surface, the longitudinal force used as the propulsion force of vehicles decreases remarkably. Thus, when running on low $\mu$ - roads, it is necessary to control the longitudinal force applied with sufficient accuracy to the front and rear wheels corresponding to the road surface so that front wheel spin may be suppressed (Fig. 6). Then, the smaller of the slip ratios of the right and left wheels of the front and rear is recognized as the slip ratio $s_{A f}$, and $s_{A r}$ which is suited to the current road surface, is given by

$s_{A f}=\frac{\left(\omega_{f} \cdot R-V\right)}{\omega_{f} \cdot R}$

and

$$
s_{A r}=\frac{\left(\omega_{r} \cdot R-V\right)}{\omega_{r} \cdot R} .
$$

Here, the driving torques, $\tau_{A f}{ }^{*}$ and $\tau_{A r}{ }^{*}$ given by (3)-(5) are adjusted so that these slip ratios may be maintained from 0.1 to 0.3 . $V$ is vehicle body speed, $R$ is radius of a tire, and $\omega_{f}$ and $\omega_{r}$ are angular speeds of the front and rear wheels, respectively.

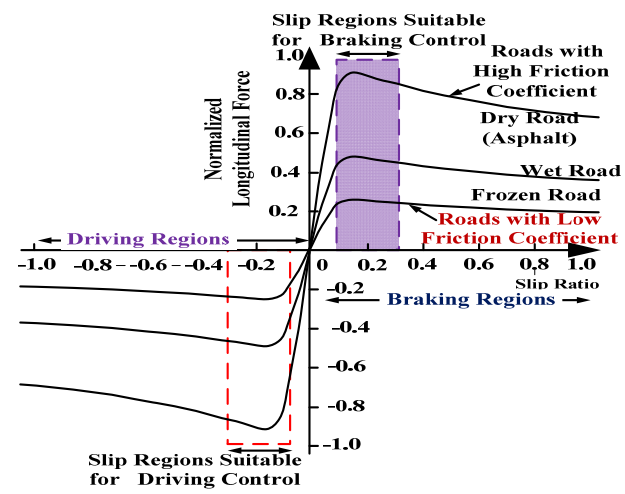

Figure 5: The stable domains of the slip ratio on the various road surfaces in driving and braking states.

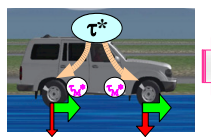

(a) Start on bad roads

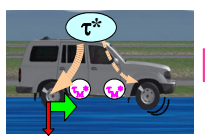

(b) Experience the front wheel

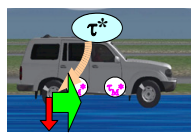

(c) Distribute the suitable driving torque to the rear wheels to suppress front wheel spin
Figure 6: Driving torque distributing method on low friction coefficient roads.

\subsection{Braking Torque Distribution Method on Low Friction Coefficient Roads}

Since the load moves to the front wheels from the rear wheels at the time of braking, rear wheels are much more apt to generate wheel lock in low $\mu$ - 
roads as compared with dry roads (Fig. 7). In order to control the wheel lock, the braking torques of the front and rear wheels are adjusted, focusing on the smaller of the slip ratios which appear in the right and left wheels of the front and rear wheels as was done for the driving regions. In this case, the braking torques given by (6)-(8) are corrected so as to always satisfy the conditions given by

$$
\begin{aligned}
& S_{B f}=\frac{\left(V-\omega_{f} \cdot R\right)}{V}, \\
& S_{B r}=\frac{\left(V-\omega_{r} \cdot R\right)}{V},
\end{aligned}
$$

and

$0.1 \leq s_{B f}, s_{B r} \leq 0.3$.

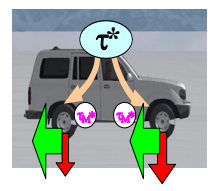

(a)Load movement and slip occur.

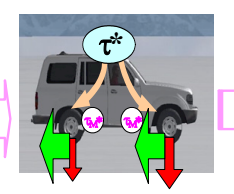

(b) Estimate the generated load movement and slip ratios

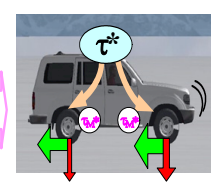

(c) Distribute proper braking torque to the front and rear wheels
Figure 7: Braking torque distribution methods when braking on low $\mu$-roads.

\subsection{A Driving Torque Distribution Method When One of the Propulsion Systems Failed}

The drive systems of the FRID EV have the failsafe drive characteristic since the front wheel drive system and the rear wheel drive system are completely independent. That is, even if one of the propulsion power systems failed, it is

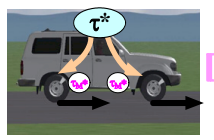

(a) Normal runs

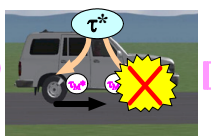

(b) Fault of a front wheel or a rear wheel drive system

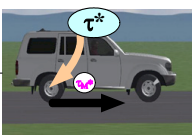

(c) Keep running with only the healthy drive system by compensating for driving torque of the failed one
Figure 8: A driving torque compensation method when the drive system of a front wheel or a rear wheel failed. possible to compensate for the driving force lost by the failure with the healthy drive system (Fig.8). Here, this compensation at the time of failure is done within limits where a healthy drive system can compensate to the utmost. With this compensation, even if the FRID EV fails during acceleration, accelerating ability is maintained within the limits of the capability of the failed vehicles.

Finally, a block diagram of the driving control system which includes the driving and braking torque distribution methods described in the above sessions is shown in Fig.9.

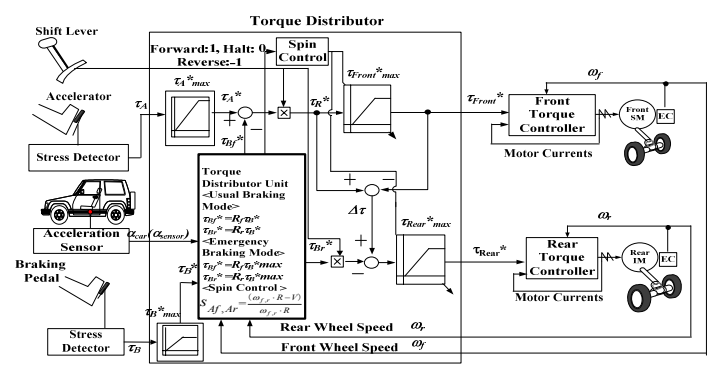

Figure 9: A driving control system including the driving and braking torque distribution methods described in the text.

\section{Verification of Running Performance of the FRID EV by Simulations}

\subsection{Effect of Driving Torque Distribution Method on Dry Roads}

The basic specifications of the drive system of the FRID EV used for the simulation are as follows. Front motor: $30 \mathrm{~kW}$ interior permanent magnet synchronous motor (IPMSM) (rated torque: 80 $\mathrm{Nm}$ ); $25 \mathrm{kw}$ induction motor (IM) (rated torque: $120 \mathrm{Nm}$ ); vehicle weight: $1850 \mathrm{~kg}$. Fig. 10 shows the effect of the driving force distribution method on dry roads when the FRID EV is accelerated to nearly $50 \mathrm{~km} / \mathrm{h}$. In Fig. 10(a), the FRID EV is being accelerated without compensation of the load movement, and a $60 \mathrm{Nm}$ torque is distributed to each of the front and rear wheels. In this case, the time to reach a velocity of $38 \mathrm{~km} / \mathrm{h}$ is about 7.5 s. On the other hand, when the driving torques of $40 \mathrm{Nm}$ and $85 \mathrm{Nm}$ are properly distributed to the front and rear wheels, in consideration of load 
movement, the acceleration time is shortened to $6.2 \mathrm{~s}$ (Fig. 10(b)). Thus, it is verified from Fig.10 that if the driving torque is distributed to the front and rear wheels in consideration of the load movement, it is possible to efficiently accelerate the FRID EV.

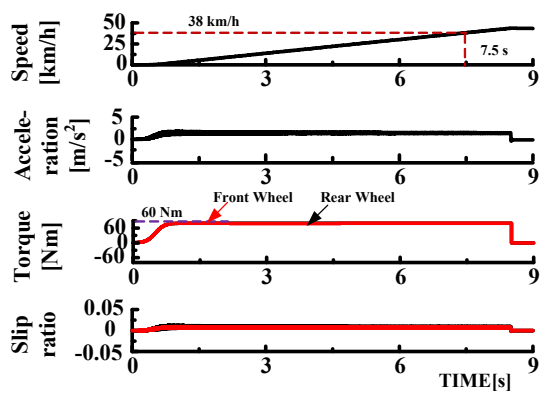

(a) Load movement not considered
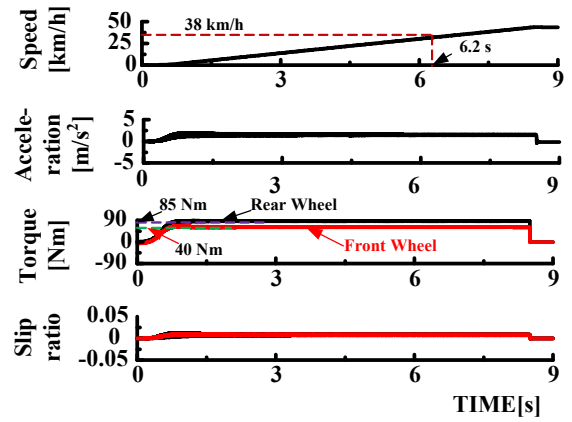

(b) Load movement considered

Figure 10: Effect of the driving torque distribution method on dry roads (high $\mu$-roads: $\mu=0.75$ ).

\subsection{Effect of the Braking Torque Distribution Method on Dry Roads}

Fig. 11 shows the effect of the braking torque distribution method on dry roads when load movement is considered. When not taking load movement into consideration, the braking torque of $60 \mathrm{Nm}$ is distributed equally to the front and rear wheels (Fig. 11(a)). When load movement is taken into consideration, the total braking torque of $120 \mathrm{Nm}$ is distributed to the front and rear wheel, and divided $80 \mathrm{Nm}$ and $40 \mathrm{Nm}$, respectively (Fig. 11(b)). It is confirmed that the braking torque distribution with consideration of the load movement shortens braking time (in the case of Fig.11: about $1 \mathrm{~s}$ ), by preventing the increase in the slip ratio of the rear wheels (Fig. 11(b)).

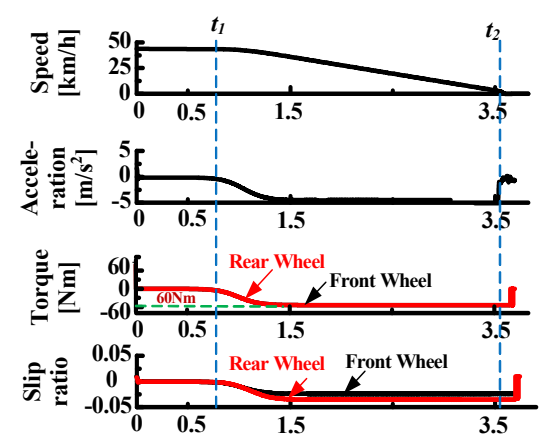

(a) Load movement not compensated

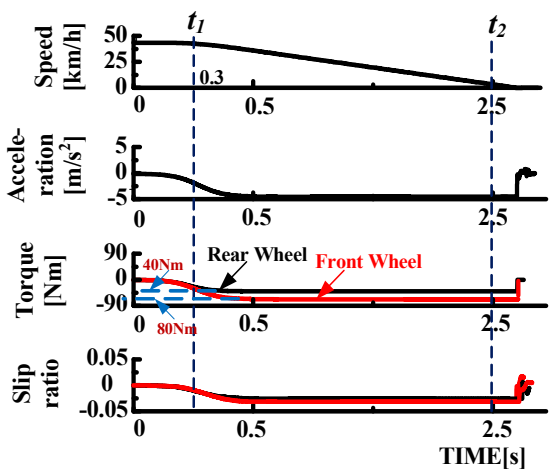

(b) Load movement compensated

Figure 11: Effect of the braking torque distribution method on dry roads (high $\mu$-roads) when load movement is considered $(\mu=0.75)$.

\subsection{Effect of the Driving Torque Distribution Method on Low Friction Coefficient Roads}

Here, the effect of the slip control in consideration of the load movement for starting on low $\mu$ - roads stably is verified. In the case of Fig. 12(a) when load movement compensation and slip control are not performed, both the front and rear wheels has caused the wheel spin at time $t_{l}$ after starting since the braking torque of $80 \mathrm{Nm}$ is distributed equally to the front and rear wheels (Fig. 12(a)). However, when the load movement compensation and slip control of front and front wheels are performed, the maximum braking torques of the front and rear wheels are corrected to proper values, i.e., to $50 \mathrm{Nm}$ and $80 \mathrm{Nm}$ which are determined based on the load movement, and then the braking torques applied to the front and rear wheels are properly adjusted while performing the slip control of both front and rear wheels within these maximums. As a result, both wheels are controlled to less than 2.5, and then the FRID EV starts without any wheel spins. 


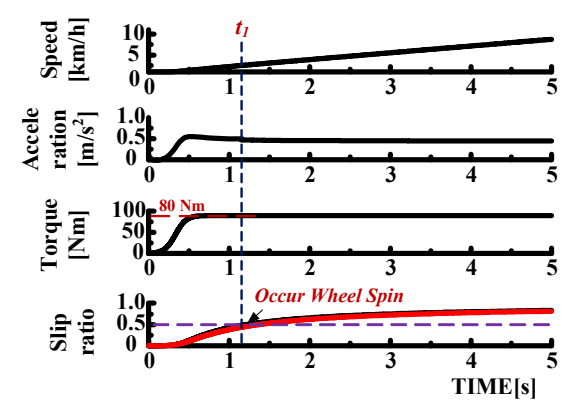

(a) Load movement compensation and the slip ratio control are not done

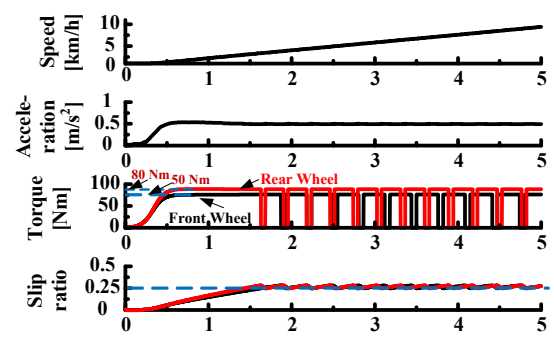

(b) Load movement compensation and the slip ratio control are done

Figure 12: Effect of driving torque distribution method on low $\mu$-roads $(\mu=0.1)$.

\subsection{Effect of Braking Torque Distribution Method on Low Friction Coefficient Roads}

If braking operation is performed on low $\mu$ roads, both the front and rear wheels can cause wheel lock easily. Then, the effect of the braking torque distribution method for controlling the wheel lock is verified. In this method, like in section 3.3, the wheel lock is controlled by performing both compensation of the load movement and slip ratio control. When the load movement compensation and slip ratio control are not carried out (Fig. 13(a)), i.e., when a constant braking torque of $120 \mathrm{Nm}$ is applied to the front and rear wheels, wheel lock occurs at almost the same time as braking operation. Then, the FRID EV keeps running under the wheel locked state until the kinetic energy of the vehicle is consumed. However, when the load movement compensation and the slip ratio control are done (Fig. 13(b)), the suitable maximum braking torque applied to the front and rear wheels is determined by taking the load movement into consideration and the slip ratio is controlled to less than 0.3 by adjusting the braking torques distributed to the front and rear wheels. As a result, drivers can stably perform braking operations on very low $\mu$-roads regardless of their individual skill.

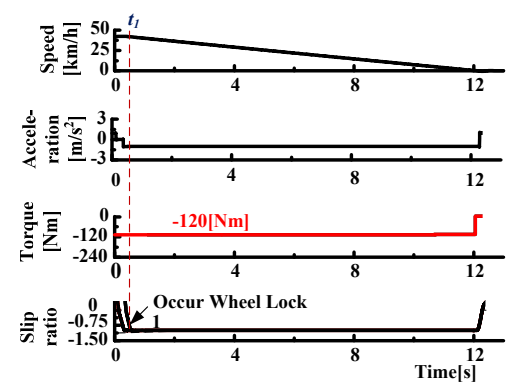

(a) Without load movement compensation and slip ratio control

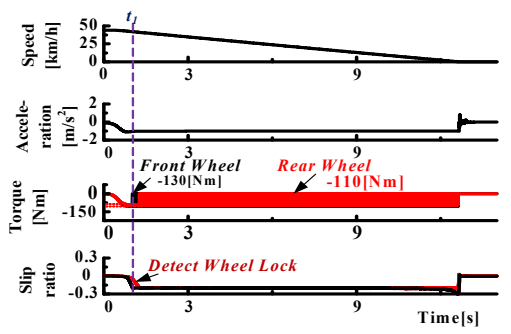

(b) With load movement compensation and slip ratio control

Figure 13: Effect of load movement compensation and slip control when braking on low $\mu$ - roads.

\subsection{Effect of the Torque Compensation at the Time of Failure}

Simulations of the dynamic characteristics at the time of failure were carried out under various running conditions. Fig.14 shows an example with and without the torque compensation at the time of failure. In Fig. 14(a), the front wheel drive system failed at time $t_{l}$. The driving torque which the front wheel drive system had generated just before failure is not compensated by the rear wheel drive system. The simulation confirms that the FRID EV cannot accelerate with the same acceleration value as before the failure appeared. During acceleration while passing another vehicle, if such a situation appears suddenly, it is extremely dangerous. However, in Fig. 14(b) at time $t_{l}$ when the front wheel drive system failed, the driving torque which the front wheel drive system had generated just before failure is compensated by the rear wheel drive system and the FRID EV maintains almost the same acceleration as before failure and can continue acceleration. It is proved that the compensation of the lost torque by the healthy driver system at the time of failure is dramatically 
effective from the viewpoint of the failsafe drive function.

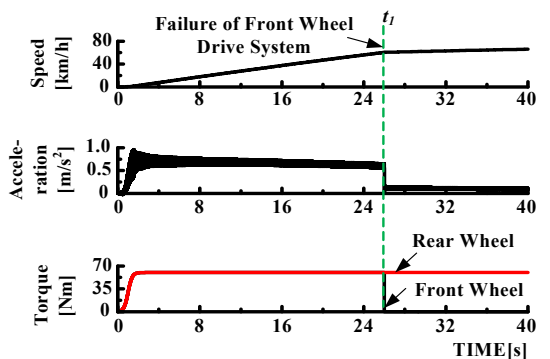

(a)Without torque compensation

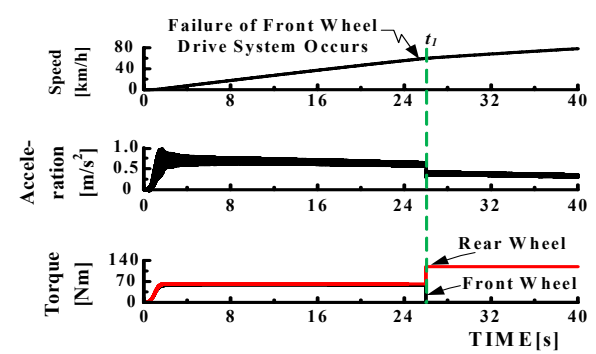

(b) With torque compensation

Figure 14: Effect of torque compensation when a front wheel drive system failed during running straight at $60 \mathrm{~km} / \mathrm{h}$, straight-propagation run.

\section{Verification of Running Performance of the FRID EV by Experiments}

\subsection{Effect of Driving Torque Distribution Method on Dry Roads}

Experiments were carried out using the prototype FRID EV (gear ratio of the front and rear wheels: 4.2 and 2.5 , respectively) which has $\mathrm{Li}$ ion batteries. In this section load movement is taken into consideration for the driving torque distributed to the front and rear wheels on dry roads. When the load movement is not considered, the front and rear tire torques, i. e., the front and rear driving torques, which are converted via differential gears of the front and rear wheels are $605 \mathrm{Nm}(=144 \times 4.2)$ and $500 \mathrm{Nm}$ $(=200 \times 2.5)$, respectively. In this case, more of the driving torque is distributed to the front wheels rather than the rear wheels (Fig. 15(a)). On the other hand, when load movement is taken into consideration, the driving torques distributed to the front and rear wheels are $412 \mathrm{Nm}$ and 500
Nm, respectively (Fig. 15(b)). It is confirmed that a larger driving torque is distributed to the rear wheels. As a result, when the energy consumption used to reach a vehicle velocity of $45 \mathrm{~km} / \mathrm{h}$ is compared between the two driving torque distribution methods, the experimental results show that it is possible to accelerate the FRID EV efficiently with about $40 \%$ less energy ( $\approx 165$ A /117 A).

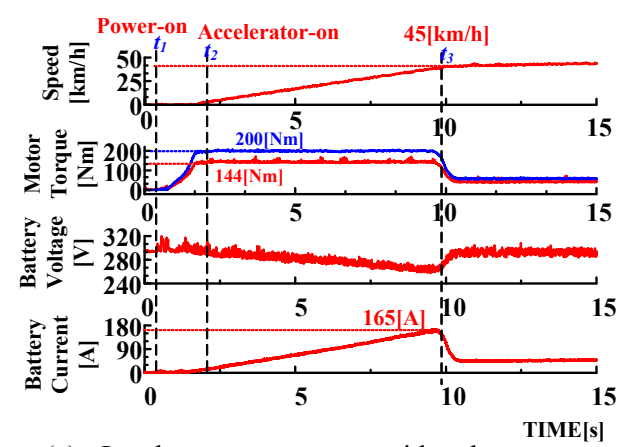

(a) Load movement not considered

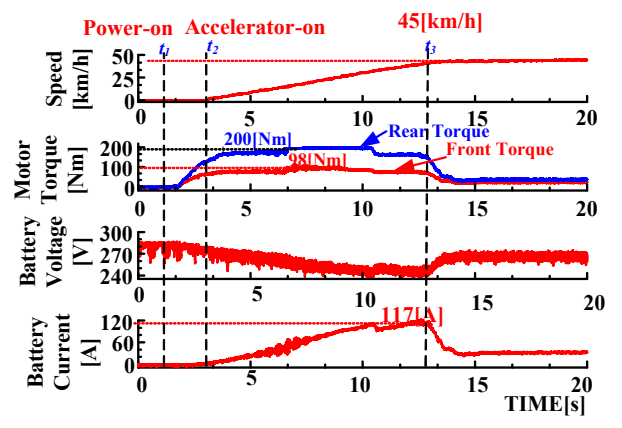

(b) Load movement considered

Figure 15: Effect of the driving torque distribution method when accelerating on dry roads while taking load movement into consideration $(\mu=0.75)$.

\subsection{Effect of the Braking Torque Distribution Method on Dry Roads}

Next, the effect of the braking torque distribution method at the time of taking the load movement into consideration was verified. When the load movement is not considered (Fig. 16(a)), the braking torques of the front and rear wheels are distributed with almost equal values, $100.8 \mathrm{Nm}$ $(=24 \times 4.2)$ and $100 \mathrm{Nm}(=40 \times 2.5)$. On the other hand, when the load movement is considered (Fig. 16(a)), the respective braking torques of $420 \mathrm{Nm}$ $(=100 \times 4.2)$ and $150 \mathrm{Nm}(=60 \times 2.5)$ are distributed to the front and rear wheels. It is confirmed that as a result of taking load movement into consideration, less braking torque is distributed to 
the rear wheels as compared with the braking torque of the front wheels. For this reason, Fig. 16(b) shows that braking force acts on the road surface effectively and the FRID EV stops in a shorter time.

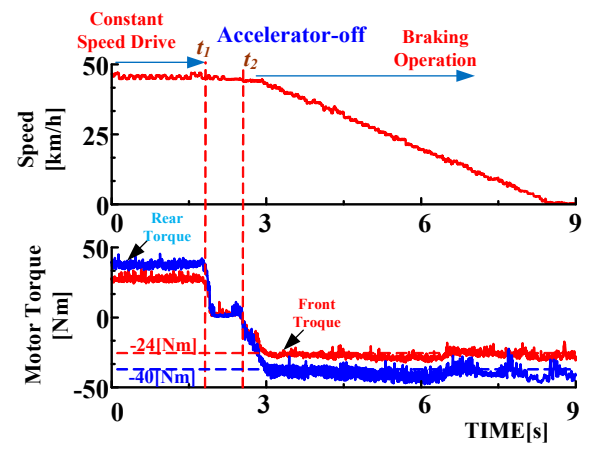

(a) Load movement not considered

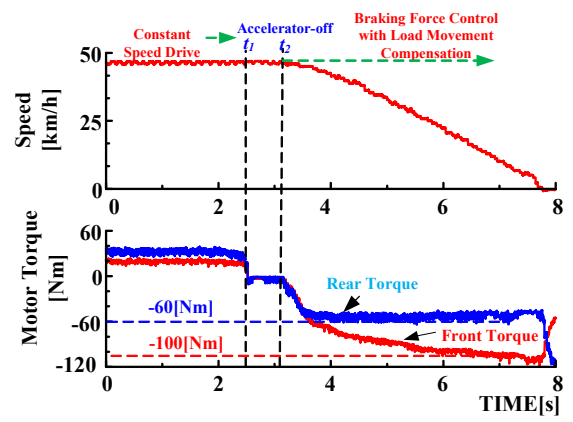

(b) Load movement is considered

Figure 16: Effect of the braking torque distribution method when braking on dry roads while taking load movement into consideration.

\subsection{Effect of Driving Torque Distribution Method on Low Friction Coefficient Roads}

The low $\mu$-road running experiments were done on a test course where the friction coefficient $\mu$ of the road surface is kept at 0.1[16]. Here, verification is made of the effect of the driving force distribution method on low $\mu$-roads when the slip ratio control in consideration of the load movement is used. When this slip ratio control is not performed (Fig. 17(a)) and the slip ratio exceeds the level of the stability limit at time $t_{l}$, the front wheel starts to experience wheel spinning so that only the speed of the front wheels increases rapidly without increasing the vehicle speed. When the slip ratio control in consideration of the load movement is used (Fig. 17(b)), since the slip ratio is controlled within the stability limit, the wheel spin is suppressed and then, the vehicle speed increases stably with the wheel speed. This means that the method is very effective when making vehicles start on very low $\mu$-roads.

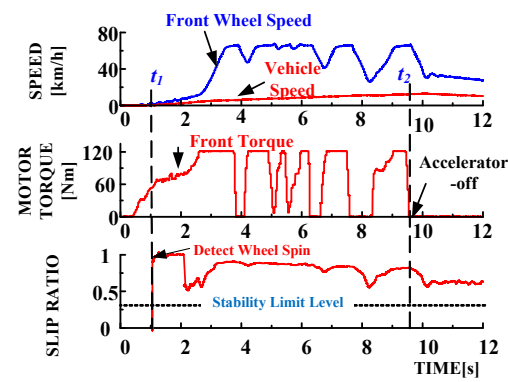

(a) Slip ratio control in consideration of the load movement is not performed.

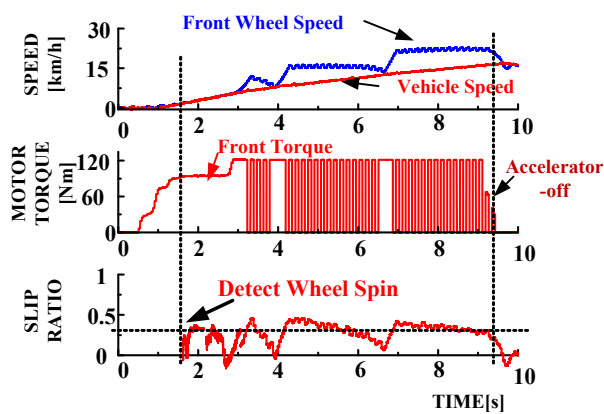

(b) Slip ratio control in consideration of the load movement is performed.

Figure 17: Effect of the driving torque distribution method on low $\mu$-roads at the time of starting $(\mu=0.1)$.

\subsection{Effect of Braking Torque Distribution Method on Low Friction Coefficient Roads}

When braking operations are performed on low $\mu$-roads, the vehicle body will glide on the road surface when the wheel becomes locked. When the slip ratio control in consideration of the load movement is not performed, after the braking operation at time $t_{l}$, the wheel lock state appears at time $t_{2}$. When the wheel lock occurs, the FRID EV is in an uncontrollable state at time $t_{2}$, and after time $t_{3}$, it keeps on gliding at almost the same speed as the speed of the front wheels (Fig. 18(a)) until the kinetic energy of the vehicle is consumed. On the other hand, when the slip ratio control is performed at time $t_{2}$ where the wheel lock was detected, the braking torques distributed to the 
front and rear wheels are adjusted so that the slip ratio becomes a stable slip ratio within the limits of maximum torque determined on the basis of load movement. As a result, the vehicle speed agrees with the front wheel speed, and stable breaking performance is obtained (Fig. 18(b)).

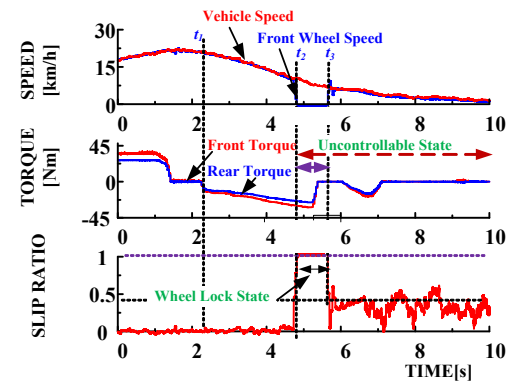

(a) Slip ratio control in consideration of the load movement is not performed.

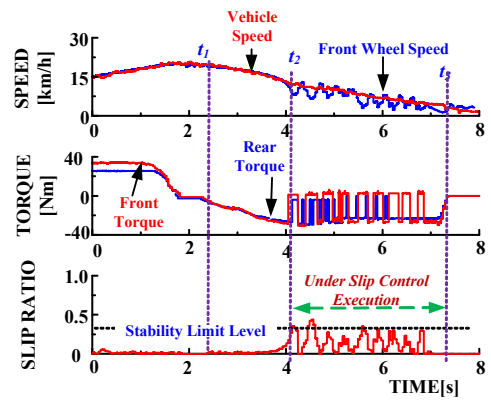

(b) Slip ratio control in consideration of the load movement is performed.

Figure18: Effect of the braking torque distribution method on low $\mu$-roads at the time of braking $(\mu=0.1)$.

\subsection{Effect of the Torque Compensation at the Time of Failure}

Finally, the fail-safe drive performance which is the most outstanding feature of FRIDEV was verified. This performance test was carried out under various running conditions. An example at the time of failure of the front drive system is shown in Fig.19. Fig. 19(a) shows drive characteristics when the lost torque of the failed front wheel is not compensated. This failure occurs at time $t_{1}$, and since the FRID EV failed in acceleration, the braking operation to stop the motion of the vehicle is done at time $t_{3}$. However, when the front wheel drive system fails at time $t_{l}$, the lost $60 \mathrm{Nm}$ torque is immediately compensated from the healthy rear wheel drive system. Then, the FRID EV normally accelerates to the velocity of $65 \mathrm{~km} / \mathrm{h}$ and braking operation for a stop is done at time $t_{3}$. The effect of compensation of the drive torque lost when there is a failure is verified.

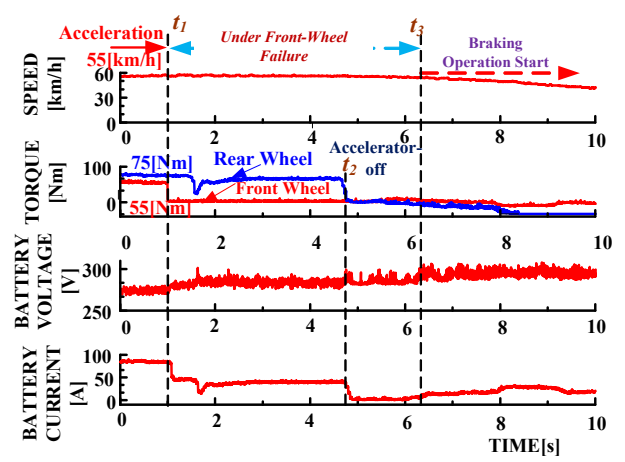

(a) When the lost torque of the failed front wheel drive system is not compensated.

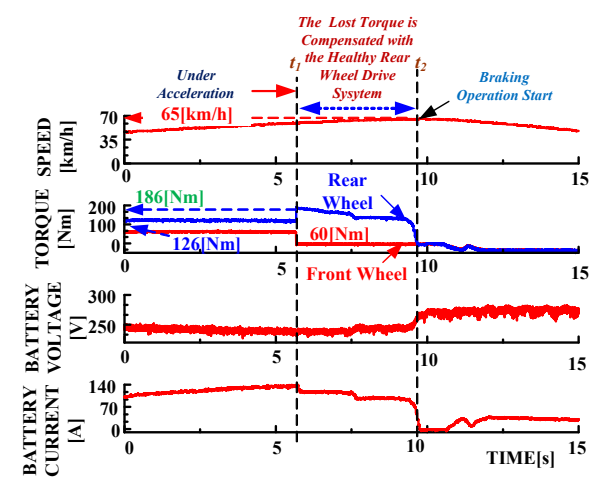

(b) When the lost torque of the failed front wheel drive system is compensated.

Figure 19: Effect of compensation of the lost torque by the rear wheel drive system when a front wheel drive system failed during acceleration at $60 \mathrm{~km} / \mathrm{h}$.

\section{Conclusions}

Since the FRID EV was proposed by Mutoh et al., as a next-generation advanced electric vehicle, its driving performance has been studied from various perspectives. Here, the safety at the time of a failure and the running performance of the FRID $\mathrm{EV}$, which are the most important issues for vehicles, were described under various running conditions. The outstanding running performance of the FRID EV was verified through simulations and experiments using the prototype FRID EV equipped with practical specifications.

All results to date indicate the FRID EV is a very promising eco-vehicle. 


\section{References}

[1] J. M. Miler, "Hybrid electric vehicle propulsion system architectures of the e-CVT type", IEEE Trans. on PLS, vol. 21, no. 3, 2006, pp. 756-767.

[2] C. C. Chan, K.T. Chau, "An overview of power electronics in electric vehicles", IEEE Trans. on IE, vol.44, no.1, 1997, pp.3-13.

[3] A. Affanni, A. Bellini, G. Franceschini, P. Guglielmi, C. Tassoni, "Battery choice and management for new-generation electric vehicles", IEEE Trans. on IE, vol. 52, no. 5, 2005, pp. 1342-1349.

[4] Y. Jeong, S. Sul, S. E. Schulz, N. R. Patel” Fault detection and fault-tolerant control of interior permanent-magnet motor drive system for electric vehicles", IEEE Trans. on IA, vol. 41, no. 1, 2005, pp. 46-51.

[5] D. Diallo, M. El H. Benbouzid, A. Makouf, "A fault-tolerant control architecture for induction motor drives in automotive applications", IEEE Trans. on VT, vol. 53, no. 6, 2004, pp. 18471855.

[6] N. Mutoh, Y. Tomita, "Failsafe Control Methods for EVs with the Failsafe Structure Driven by Front and Rear Wheels Independently", The World Electric Vehicle Association Journal, vol.1,2007, pp.271-278.

[7] Mitsubishi innovative Electric Vehicle, "iMiEV", http://www.mitsubishimotors.co.jp/corporate/technology/environment/ miev.html

[8] A. Kawamura, N. Hoshi, T. W. Kim. T. Yokoyama, T. Kume, "Analysis of antidirectional-twin-rotary motor drive characteristics for electric vehicles", IEEE Trans. on IE, vol. 44, 1997, pp 64-70.

[9] Mitsubishi Goes electric: New EVs in 2010, MIEV (Mitsubishi In-wheel motor electric vehicle; "Colt EV for test vehicle", http://www.greencarcongress.com/2005/05/mits ubishi goes.html, 2005 Automotive Engineering Exposition, May 18-20, 2005, Yokohama, Japan.

[10] M. Terashima, T. Ashikaga, T. Mizuno, K. Natori, N. Fujiwara, M. Yada, "Novel Motors and Controllers for High Performance Electric Vehicle with Four In-Wheel Motors," IEEE Trans. on IE, vol. 44, no. 1, 1997, pp. 28-38.

[11] N. Mutoh, Y. Miyazaki, R. Masaki, F. Tajima, T. Ohmae, "Electric Drive System and Drive Method", U.S Patent 5549172, 1996.

[12] N. Mutoh, T. Kazama, K. Takita, "Driving characteristics of an Electric Vehicle System with Independently Driven Front and Rear Wheels ", IEEE Trans. on IES, vol.5 3, no. 3, 2006, pp. $803-813$.

[13] N. Mutoh, Y. Takahashi, Y. Tomita," Failsafe Drive Performance of FRID electric vehicles
With the structure driven by the Front and Rear Wheels Independently" IEEE Trans. on IE, vol. 55, no. 6, 2008, pp. $2306-2315$.

[14] N. Mutoh, Y. Hayano, H. Yahagi, K. Takita, "Electric braking control methods for electric vehicles with independently driven front and rear wheels" IEEE Trans. on IES, vol. 54, no.2, 2007, pp. 1168-1176.

[15] R. Rajamani, "Vehicle dynamics and control", Mechanical Engineering Series, Springer Science, New York, 2006, pp. 137-151.

[16] Japan Automobile Research Institute, Running Test Centre, http://www.jari.or.jp/index.html

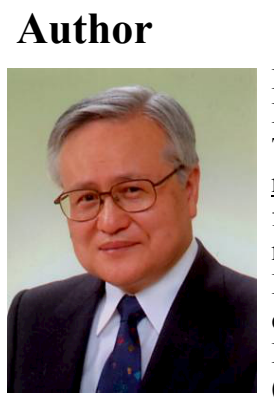

Dr. Nobuyoshi Mutoh Professor, Graduate School of Tokyo Metropolitan University, nmutoh@cc.tmit.ac.jp. Major fields are advanced echomachine control systems such as EVs. PV, wind power and fuel cells. IEEE senior member, Professional Engineer (Electronics and Electronics) 